References

Feltkamp-Vroom, T. M. (1975). Substrates and their processing: preparation of tissues and cells for immunohistochemical processing. Annals of the New York Academy of Sciences, 254, 21-26.

Nairn, R. C. (Ed) (1969). Immunological tracing: general considerations. In Fluorescent Protein Tracing, 3rd edition, Chapter 6, pp. 111-151. Livingstone, Edinburgh.

Pearse, A. G. E. (1968). Cold knife and cold microtome (Cryostat) methods. In Histochemistry, Theoretical and Applied, 3rd edition, Vol. 1, Chapter 2, pp. 13-26. Little, Brown, Boston.

\section{Application of a modified Attwood's stain to the study of decalcified bone sections}

S. PUTNS AND D. J. DESA Department of Pathology, McMaster University Medical Center, 1200 Main St. West, Hamilton, Ontario, Canada (L8S 4J9)

Using routine histological procedures, it is difficult to distinguish osteoid from calcified bone and woven from lamellar bone in decalcified preparations of bone. Although several alternatives are available to overcome the first difficulty, differential staining of woven and lamellar bone has not been described.

In 1958, Attwood modified Lendrum's (1947) phloxine-tartrazine stain to identify fetal amniotic squames in maternal pulmonary vessels. During a study of the organisation of amniotic debris in the middle ear cavity of newborn infants (deSa, 1977) it was noted that the osseous components of the middle ear and the petrous temporal bone were staining in an irregular fashion. By examination of the sections with polarised light it was seen that the 'lamellar' bone stained selectively with phloxine. It was decided to investigate this phenomenon further.

\section{Material and methods}

Formalin-fixed, paraffin-embedded sections from patients with osteoid osteoma, osteogenic sarcoma, Paget's disease of bone, fracture callus, subperiosteal fibrous defect (periosteal desmoid), and costochondral junctions from infants with severe growth retardation and from infants with older pseudorachitic changes were selected. They were stained with a modification of alcian green, phloxine, and tartrazine (Attwood's stain) using prior oxidation with $0.5 \%$ potassium permanganate (Table). Phloxine B solutions were prepared both with and without

Received for publication 28 March 1977 calcium chloride. Some sections were treated with periodic acid before staining.

\section{Results}

In sections stained with prior oxidation with potassium permanganate and stained with phloxine $B$ solution containing calcium chloride, a clear distinction between the phloxine positive lamellar bone and the tartrazine positive woven bone was demonstrated (Figs 1 to 4). In particular, the nidus of an osteoid osteoma could be clearly distinguished from the surrounding sclerotic bone, and fracture callus with its woven bone could be readily distinguished from the mature bone of the cortex of a rib. In all cases, cartilage present in the section stained strongly with alcian green.

Differential staining was altered if calcium chloride was omitted from the phloxine B solution. In these sections even the lamellar bone lost much of its affinity for phloxine. Pretreatment of sections with periodic acid resulted in all bone, whether woven or lamellar, staining positively with phloxine.

In other randomly selected bone specimens, it was noted that the osteoid seams on the growing surface of bone trabeculae were stained preferentially with tartrazine and could be distinguished from the older lamellar layers of bone which stained selectively with phloxine (Fig. 1).

\section{CHANGING STAINING CHARACTERISTICS OF MATURING BONE}

It became apparent that in older areas of fracture callus and in the central areas of some tumours, the staining characteristics of woven bone changed. An increasing number of phloxine-positive areas could be identified in the maturing segments of bone, and as callus matured its staining characteristics could be seen to change dramatically. This feature could be used as an extremely valuable control of the relative sensitivity of the staining technique, if appropriate sections were selected as a control. 
Table Modified Attwood's stain

Solutions required:

\begin{tabular}{lll}
\hline $1 \%$ Alcian green & $\begin{array}{l}\text { Alcian green } 2 \mathrm{GX} \text { (Gurr) } \\
\text { Glacial acetic acid } \\
\text { Distilled water }\end{array}$ & $\begin{array}{r}1 \mathrm{~g} \\
2 \mathrm{~m}\end{array}$ \\
& $98 \mathrm{~m}$
\end{tabular}

$2 \quad 0.5 \%$ Potassium permanganate solution

$35 \%$ Oxalic acid solution

4 Harris' haematoxylin solution Haematoxylin (Gurr) $1 \mathrm{~g}$

Potassium alum $\quad 20 \mathrm{~g}$

Absolute alcohol $\quad 10 \mathrm{ml}$

Distilled water $\quad 200 \mathrm{ml}$

(To above solution add $0.5 \mathrm{~g}$ mercuric chloride)

5 Acid alcohol (1\% w/v concentrate-hydrochloric acid in $70 \%$ ethyl alcohol)

6 Scott's tap water substitute Sodium bicarbonate $3.5 \mathrm{~g}$

Magnesium sulphate $20 \mathrm{~g}$

$7 \quad 0.5 \%$ Phloxine B

Distilled water $\quad 1000 \mathrm{ml}$

Phloxine B (Gurr, CI 45410) $0.5 \mathrm{~g}$

Calcium chloride $0.5 \mathrm{~g}$

Distilled water $\quad 100 \mathrm{ml}$

8 Ethylene glycol monoethyl ether (cellosolve) [Fischer]

$92 \%$ Tartrazine Tartrazine (Gurr, CI 19140) $2 \mathrm{~g}$

Cellosolve $1000 \mathrm{ml}$

[Solution heated in a water bath to form a saturated solution: cooled and filtered before use]

Staining procedure:

1 Sections taken down to water in the usual manner

2 Stained in $1 \%$ Alcian green for 5 minutes

3 Rinsed in tap water

4 Treated with $0.5 \%$ potassium permanganate for 2 minutes

5 Rinsed in tap water

6 Decolourised in $5 \%$ oxalic acid solution for 5 minutes

7 Washed in tap water

8 Stained in Harris' haematoxylin for 2-5 minutes

9 Rinsed and developed in tap water

10 Differentiated in $1 \%$ acid alcohol

11 Rinsed in tap water and then developed in Scott's tap water substitute for 5 minutes

12 Rinsed in tap water

13 Stained in phloxine solution for 15-30 minutes

14 Rinsed in tap water and cellosolve

15 Differentiated in tartrazine-cellosolve for 30 seconds to one minute with agitation of slide-tray

16 Dehydrated in two changes of cellosolve

17 Cleared in xylol and mounted in permount

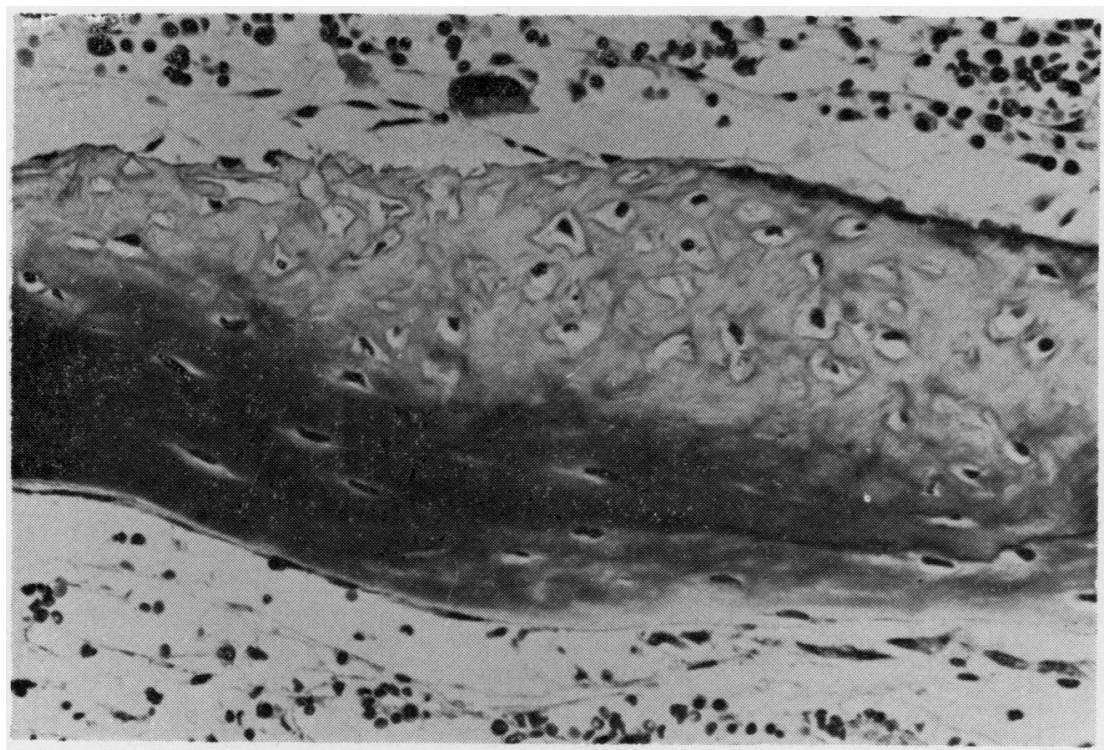

Fig. 1 A bony trabecula from the diploe of an infant with growth retardation showing $a$ broad band of darkly stained phloxinophilic bone capped by an area of bone that is stained lightly with tartrazine. Note also the thin rim of newly deposited bone, staining selectively with tartrazine on the lower surface of the trabecula (Modified Attwood $(M A)$ stain $\times 200)$. 


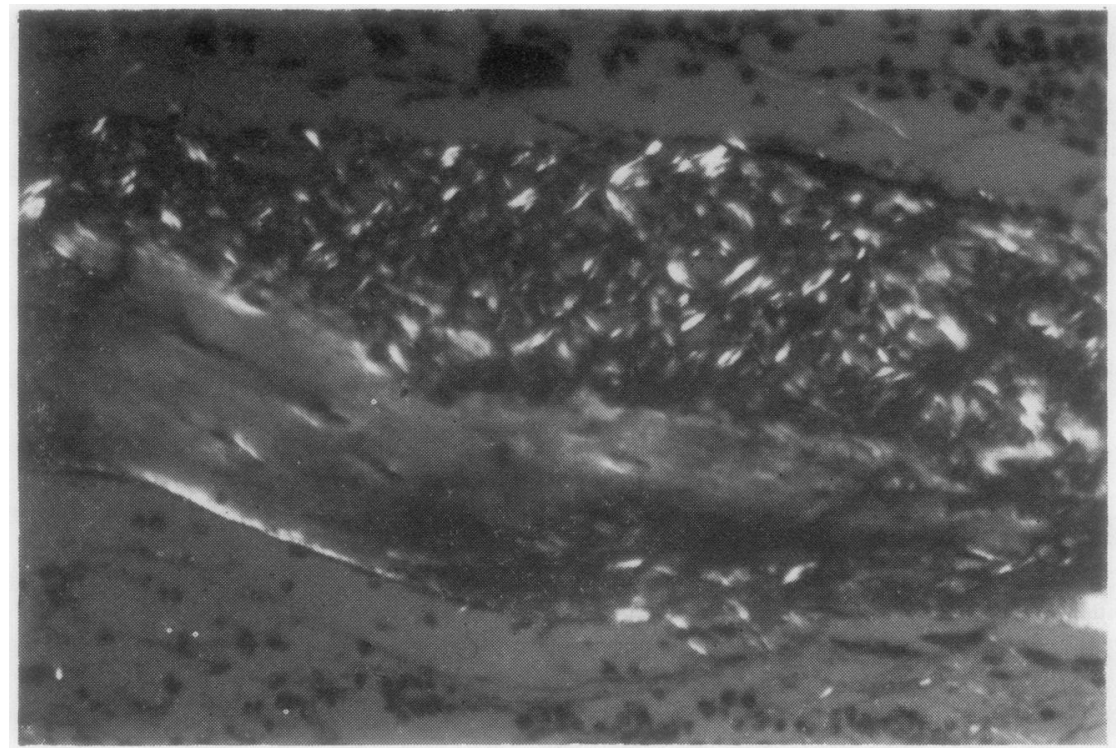

Fig. 2 Same field as in Fig. 1 viewed in polarised light, showing that the phloxinophilic areas of the bone correspond to lamellar bone, and that the areas stained with tartrazine represent newer bone. Note also the small 'transitional' area in the lower right segment of the trabecula $(M A$, polarised light $\times 200)$.

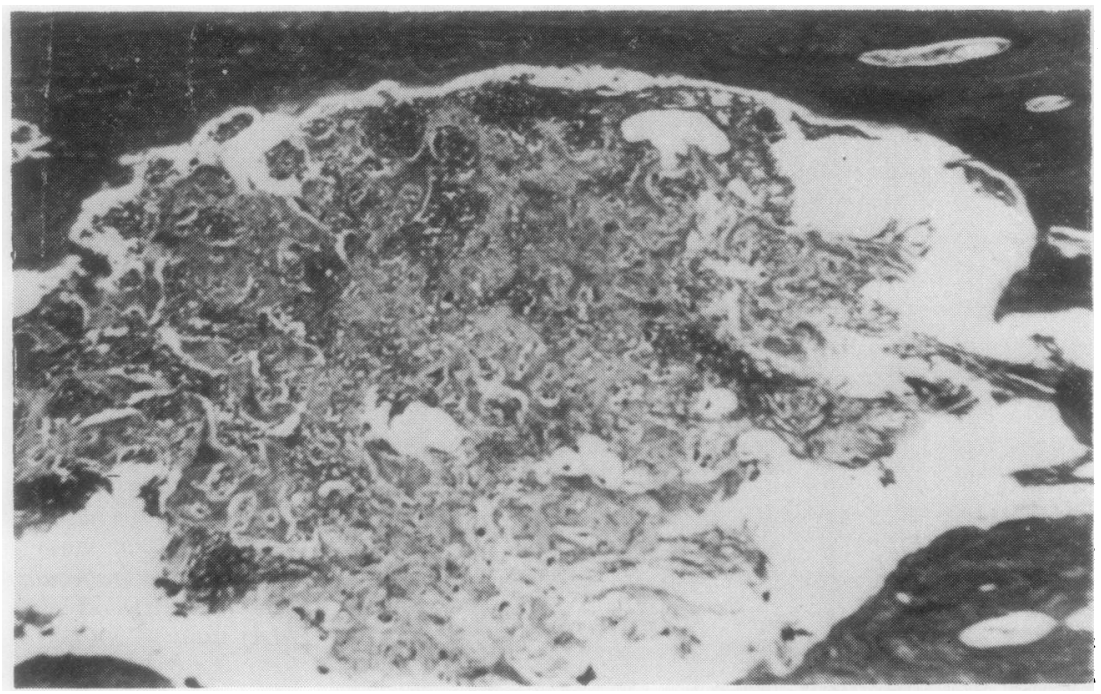

Fig. 3 The bone in the nidus of this osteoid osteoma stains differentially with tartrazine while the sclerotic surrounding bone stains selectively with phloxine $(M A \times 30)$. 


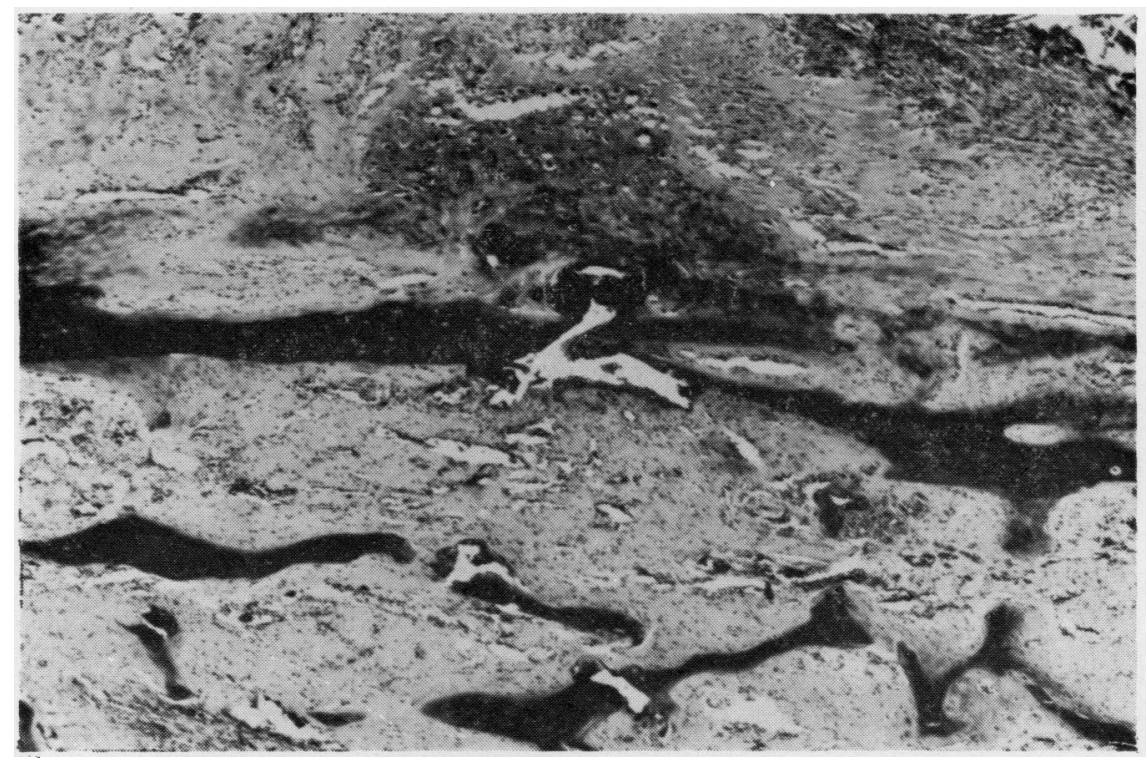

Fig. 4 A fracture callus shows the tartrazine-staining newly formed bone of the callus, both within the medulla and subperiosteally, with an obvious distinction from fractured trabecula of phloxinophilic (darkly staining) bone. Note the cartilaginous nodules that stain positively with alcian green $(M A \times 30)$.

\section{Comment}

The particular staining technique used in this report appears to have an advantage over the routine haematoxylin-eosin stain preparation. The obvious distinction between woven and lamellar bone facilitates the study of bone lesions. This could prove to be of considerable use in any morphometric study of bone disease, as well as in the preparation of material for teaching purposes.

\section{References}

Attwood, H. D. (1958). The histological diagnosis of amniotic fluid embolism. Journal of Pathology and Bacteriology, 76, 211-215.

deSa, D. J. (1977). Polypoidal organization of aspirated amniotic squamous debris (amnion nodosum) in the middle-ear cavity of newborn infants. Archives of Disease in Childhood, 52, 148-151.

Lendrum, A. C. (1947). The phloxine-tartrazine method as a general histological stain and for the demonstration of inclusion bodies. Journal of Pathology and Bacteriology, 59, 399-404. 